Journal for ImmunoTherapy of Cancer

\title{
Timing of steroid initiation and response rates to immune checkpoint inhibitors in metastatic cancer
}

Diana V Maslov (D) , ${ }^{1}$ Karine Tawagi, ${ }^{2}$ Madhav KC, ${ }^{3}$ Victoria Simenson, ${ }^{1}$ Helen Yuan, ${ }^{1}$ Cameron Parent, ${ }^{1}$ Adi Bamnolker, ${ }^{1}$ Richa Goel, ${ }^{1}$ Zoe Blake, ${ }^{4}$ Marc R Matrana, ${ }^{5}$ Daniel H Johnson ${ }^{5}$

To cite: Maslov DV, Tawagi K, $\mathrm{KC} \mathrm{M}$, et al. Timing of steroid initiation and response rates to immune checkpoint inhibitors in metastatic cancer. Journal for ImmunoTherapy of Cancer 2021;9:e002261. doi:10.1136/ jitc-2020-002261

Accepted 17 May 2021
Check for updates

(C) Author(s) (or their employer(s)) 2021. Re-use permitted under CC BY-NC. No commercial re-use. See rights and permissions. Published by BMJ.

${ }^{1}$ Internal Medicine, Ochsner Medical Center-New Orleans, New Orleans, Louisiana, USA ${ }^{2}$ Hematology/Oncology, Ochsner Medical Center-New Orleans, New Orleans, Louisiana, USA

${ }^{3}$ Public Health, Louisiana State University Health Sciences Center, New Orleans, Louisiana, USA

${ }^{4}$ Medical School, The University of Queensland School of

Medicine, Herston, Queensland, Australia

${ }^{5}$ Hematology/Oncology, Ochsner Cancer Institute, New Orleans, Louisiana, USA

Correspondence to

Dr Diana V Maslov;

diana.maslov@ochsner.org

\section{ABSTRACT}

Background Corticosteroids (CS) are the mainstay of immune-related adverse effect (irAE) management, as well as for other indications in cancer treatment. Previous studies evaluating whether CS affect immune checkpoint inhibitor (CPI) efficacy compared patients receiving CS versus no CS. However, there is a paucity of clinical data evaluating the timing of concomitant CS and CPI efficacy. Methods We retrospectively collected data from patients who received CS during CPI treatment at a single institution. Patients were in two cohorts based on timing of initiation of CS ( $\geq 2$ months vs $<2$ months after initiating CPI). Patient characteristics, irAEs, cancer type, treatment type, treatment response/progression per RECIST V.1.1, and survival data were collected. Kaplan-Meier and Cox proportional hazard regression methods estimated HRs for the primary endpoint of progression-free survival (PFS) along with overall survival (OS).

Results We identified 247 patients with metastatic cancer who received CS concurrently with CPIs. The median time on CS was 1.8 months. After adjusting for treatment type, tumor type, brain metastases, and irAEs, those treated with $\mathrm{CS} \geq 2$ months after starting CPI had a statistically significant longer PFS (HR=0.30, $p<0.001)$, and OS (HR $0.34, \mathrm{p}<0.0001$ ) than those who received $\mathrm{CS}<2$ months after starting CPI. Objective response rate (ORR) for patients on CS $\geq 2$ months was $39.8 \%$, versus ORR for patients $<2$ months was $14.7 \%$ ( $p$ value $=<0.001$ ) Conclusion Our results suggest that early use of CS during CPI treatment significantly hinders CPI efficacy. This data needs to be validated prospectively. Future studies should focus on the immune mechanisms by which CSs affect T-cell function early in the CPI treatment course.

\section{BACKGROUND}

Since the Food and Drug Association (FDA) approval of ipilimumab in 2011, immune checkpoint inhibitors (CPIs) have revolutionized the cancer treatment landscape, and are now being studied in most tumor types, both in the metastatic and early stage setting. ${ }^{1}$ Because CPIs dysregulate physiologic mechanisms of T-cell tolerance, patients can experience immune-related adverse effects (irAEs), for which the first-line management often consists of corticosteroids (CS).$^{2-7}$ In addition, CS are often used as palliative treatment for cancer-related symptoms such as anorexia, nausea prevention, dyspnea, fatigue, and symptomatic brain metastases. ${ }^{8-11}$ For many CPI trials, a threshold of $\geq 10 \mathrm{mg}$ of prednisone is a key exclusion criteria, because of concerns of the immunosuppressive effects on CPI efficacy. ${ }^{12}$

The majority of retrospective data evaluating CS effects on cancer immunotherapy efficacy have found that CS does not hinder CPI outcomes. ${ }^{13-17}$ These studies compared patients receiving CS versus no CS, which may be confounded by higher rates of irAEs in patients that receive CS and a known association of irAEs with higher response rates to CPIs. There have been other retrospective studies demonstrating negative effects of early and baseline CS on CPI efficacy. ${ }^{18-22}$ A large systematic review and meta-analysis found that a negative impact of CS use was observed for both overall survival (OS) and progression-free survival (PFS) in patients that received CS for supportive care or brain metastases. CS used to mitigate irAEs did not negatively affect outcomes, but this study again was comparing CS versus no CS treatment. ${ }^{18}$ Similar findings have been described in a population of patients with non-small cell lung cancer (NSCLC) treated with single-agent Program Death Ligand 1 (PD(L)1) blockade, where baseline CS use of $\geq 10 \mathrm{mg}$ was associated with poorer outcomes in patients treated with CPIs compared with patients that did not receive CS. The most common indications for CS in these studies were dyspnea, fatigue, and brain metastases-factors that would likely affect prognosis negatively compared with patients that are not on baseline CS prior to CPI initiation. ${ }^{19}$ Based on these data, it is difficult to 
make conclusions regarding the direct effects of CS on CPI efficacy.

\section{METHODS \\ Patients}

This retrospective cohort study included patients with metastatic cancer who presented to the Ochsner Cancer Institute and received CS during their CPI treatment between October 1, 2014 and January 21, 2020. Data were gathered through the electronic medical record. A total of 600 patients were captured who had a cancer diagnosis and received both CS and immunotherapy. Patients who received at least one cycle of Program Death-1/Program Death-Ligand1 (PD-1/PD-L1) immunotherapy (nivolumab, pembrolizumab, atezolizumab, or durvalumab) with or without the combination of Cytotoxic T-Lymphocyte-Associated Protein 4 (CTLA-4) inhibitor, ipilimumab, during the study period were eligible. Patients who did not receive CS while on immunotherapy or those who did not undergo re-staging imaging while on treatment were excluded. Patients who received chemotherapy concurrently with immunotherapy were excluded. IrAEs were defined using the Common Terminology Criteria for Adverse Events version 5 (CTCAEv5) criteria.

Demographics (age, gender, race, smoking history), Eastern Cooperative Oncology Group (ECOG) Performance Status at the start of treatment, imaging results (CT scans, MRI, positron emission tomography), time on treatment, best response to CPI, date of progression of disease, prevalence and type of irAE while on CPI, prednisone equivalent dosing, and date of steroid initiation while on CPI were collected. Patient's smoking status was also collected. Smoking status was categorized as light smoker $(<10$ pack years) and heavy smoker ( $\geq 10$ pack years)

\section{Statistical analysis}

Bivariate analyses were performed between time on CS ( $\geq 2$ months or $<2$ months) with patients' demographics, medical history, and clinical outcomes. For categorical data, $\chi^{2}$ or Fisher-exact test was used to compare the groups, and results are reported as frequencies and percentages. For continuous variables, independent sample t-test or Mann-Whitney $\mathrm{U}$ test was used to compare patient groups. SAS V.9.4 for windows was used to conduct all statistical analyses. Tests were performed at a significance level of $\alpha=0.05$. Values were considered statistically significant if $\mathrm{p}$ value was less than 0.05 .

The primary endpoint is PFS with key secondary endpoints of OS and objective response rate (ORR). ORR and progression were defined per Response Evaluation Criteria in Solid Tumors version 1.1 (RECIST v 1.1). Kaplan-Meier and Cox proportional hazard regression methods were used to estimate the survival probability and Hazard Ratios (HRs). Crude and adjusted HRs of progressive disease and mortality were calculated. CS for brain metastases, irAEs, cancer type, and drug type were identified as potential confounders and were adjusted in the final (adjusted) Cox proportional hazard regression model.

\section{RESULTS}

\section{Patient characteristics}

We identified 247 patients with metastatic cancer who received CS for irAE who met the study criteria. The median age of patients was 69 years. The median time on CS for all patients was 1.8 months. There were 24 patients that were on baseline CS. Demographic characteristics between those who received CS $\geq 2$ months after CPI initiation and those who received CS $<2$ months after CPI initiation were similar between the two groups for gender, smoking, ECOG, body mass index, but different for age and race $(\mathrm{p}=0.04)$ (table 1$)$.

The majority of patients who received CS had NSCLC $(\mathrm{n}=98,40 \%)$, followed by renal cell carcinoma $(\mathrm{n}=43$, $17.40 \%)$, and melanoma $(n=30,12.5 \%)$. In terms of treatment type, most patients were on nivolumab $(\mathrm{n}=168$, $68.0 \%)$, and pembrolizumab ( $\mathrm{n}=61,25.7 \%)$ immunotherapy and a total of $195(79.0 \%)$ patients received ipilimumab either alone or in combination with a PD-1/ PD-L1 inhibitor (table 1).

Of 247 patients, a total of $137(55.5 \%)$ had an irAE. Of these patients with irAEs, $51.8 \%(\mathrm{n}=71)$ started CS $\geq 2$ months after immunotherapy and $48.1 \% \quad(n=66)$ started CS $<2$ months after immunotherapy. In the study population, the most common adverse effects were thyroiditis $(\mathrm{n}=39,15.8 \%)$, dermatitis $(\mathrm{n}=38,15.4 \%)$, and colitis $(38,15.4 \%)$. Among the $\geq 2$ months CS group, the most common irAEs were thyroiditis $(64.5 \%, \mathrm{n}=25)$, and colitis (55.2\%, $\mathrm{n}=21$ ). Among those that started CS $<2$ months, dermatitis $(60.5 \%, \mathrm{n}=23)$ was the most common followed by pneumonitis $(55.8 \%, \mathrm{n}=19)$ (table 2 ).

\section{Progression-free survival}

For PFS, the median follow-up time for those on CS $\geq 2$ months after CPI initiation was 24.5 months, and the median follow-up time CS $<2$ months after CPIs was 22.9 months. CSs received $\geq 2$ months after initiation of immunotherapy were associated with an increased PFS compared with patients who received CS $<2$ months (figure 1; median PFS 14.9 vs 2.8 months; adjusted $\mathrm{HR}=0.30$; $\mathrm{p}$ value $<0.0001$ ). This association was significant both after adjusting for confounding factors such as brain metastases, irAEs, cancer type, drug type and unadjusted using a multivariable analysis (adjusted HR and unadjusted HR). Steroid dosage was also evaluated. We compared those patients who had received $\geq 1000 \mathrm{mg}$ of prednisone equivalents to those who received $<1000 \mathrm{mg}$ of prednisone equivalents while receiving CPIs. There was not a significant relationship between the dose of CS received while on CPIs and $\mathrm{PFS}(\mathrm{HR}=1.10, \mathrm{p}=0.55)$.

\section{Overall survival}

For OS, the median follow-up time for those on CS $\geq 2$ months after CPI initiation was 26.8 months, and the median follow-up time CS $<2$ months after CPIs was 
Table 1 Demographics characteristics of patients by steroids timing $(n=247)$

\section{Patient's characteristics}

Median age (IQR), overall: 69

Gender

Male

Female

Race

$\begin{array}{lr}\text { Black } & 28(53.85) \\ \text { White } & 99(54.10) \\ \text { Others } & 2(16.67)\end{array}$

Smoking

$\begin{array}{ll}\text { Never/light smoker } & 59(57.28) \\ \text { Heavy smoker } & 69(48.2) \\ \text { ECOG grade } & \end{array}$

Grade 0

Grade 0

Grade 1

Grade 2

Grade 3

BMI

Underweight

Normal

Overweight

Obese

Brain metastases

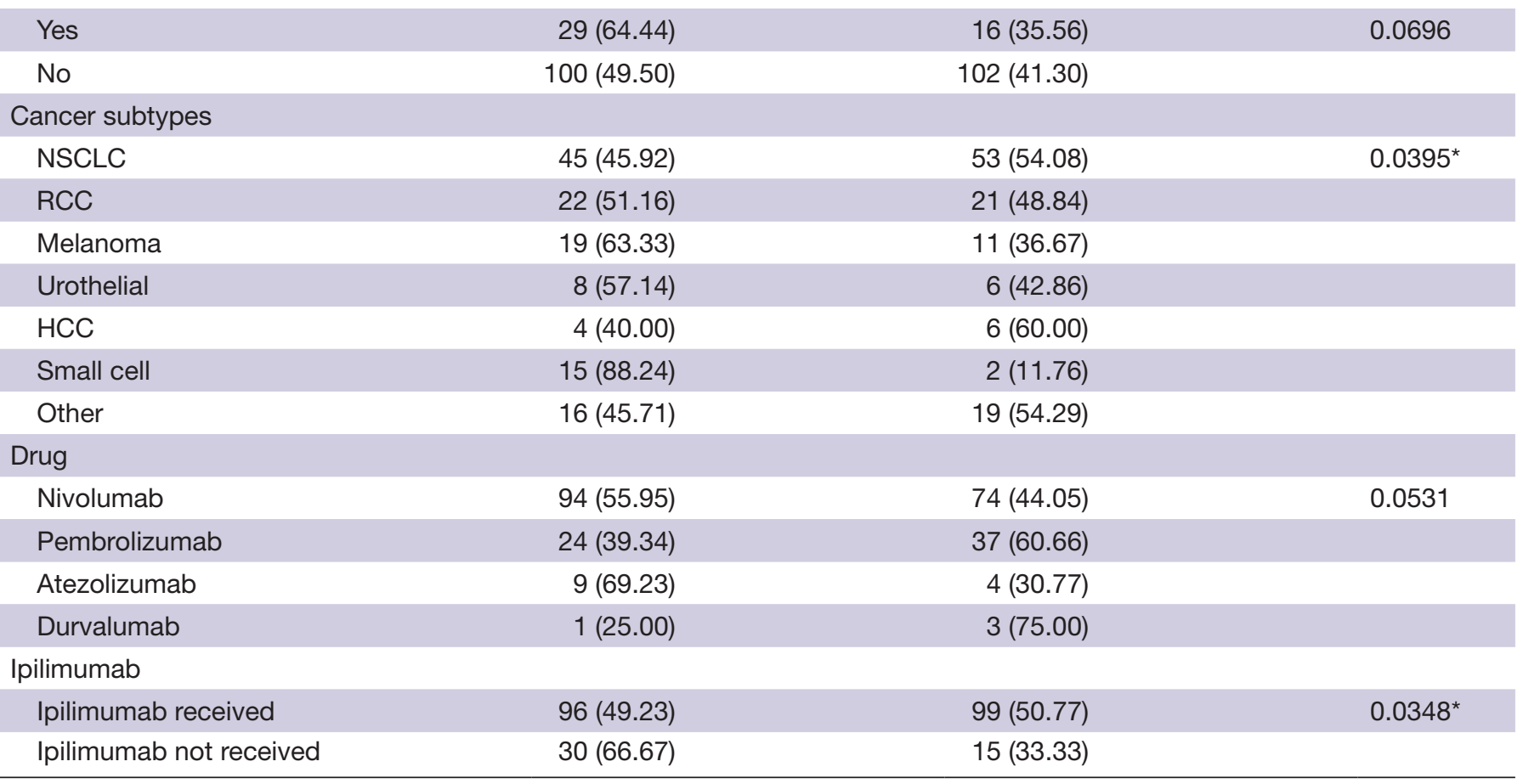

*Statistically significant at 0.05 .

BMI, body mass index; ECOG, Eastern Cooperative Oncology Group; HCC, Hepatocellular Carcinoma; NSCLC, non-small cell lung cancer; RCC, Renal Cell Carcinoma.

\begin{tabular}{ll} 
Steroids $\geq \mathbf{2}$ months & P value \\
\hline $71(15)$ & 0.0048
\end{tabular}

$78(47.27)$

0.8232

40 (48.78)

24 (46.15)

$0.04^{*}$

84 (45.90)

10 (83.33)

44 (42.72)

0.1619

74 (51.75)

43 (48.86)

0.3242

56 (52.83)

16 (36.36)

28 (63.63)

3 (50.00)

15 (51.72)

14 (48.28)

0.4375

56 (26.67)

41 (42.27)

30 (51.72)

28 (48.28)

28 (21.71)

35 (55.56)

16 (35.56) 
Table 2 Immune-mediated adverse effect (AE) by steroids timing $(n=247)$

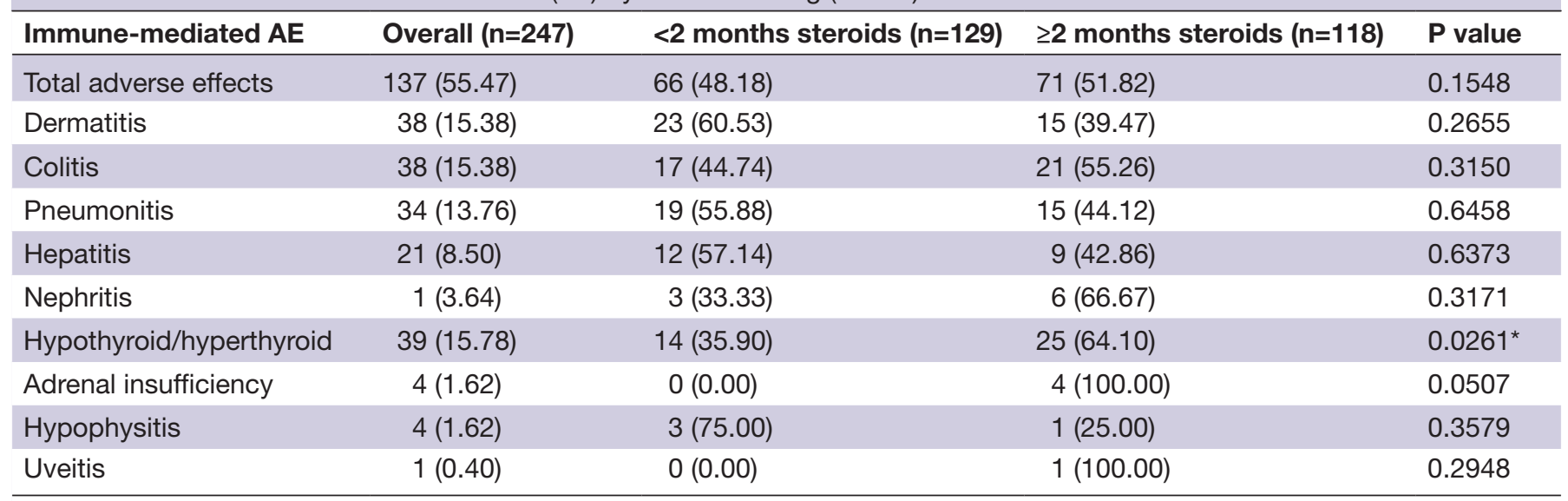

*Statistically significant at 0.05 .

25.0 months. CS received $\geq 2$ months after initiation of immunotherapy were also associated with an increased OS compared with patients who received $\mathrm{CS}<2$ months (figure 2; median OS 25.0 vs 6.4 months; adjusted $\mathrm{HR}=0.34 ; \mathrm{p}$ value $<0.0001)$. This was also adjusted for brain metastases, irAE, cancer type, and drug type (adjusted and unadjusted HR). There was not a significant relationship between the dose of CS received while on CPIs and OS as seen with the PFS analysis $(\mathrm{HR}=1.15$, $\mathrm{p}=0.38)$.

\section{Therapeutic response}

In the total population, the overall response rate was $26.7 \%$ (66 patients). The $\geq 2$ month group had an ORR of $39.8 \%(\mathrm{n}=118)$ while the $<2$ month group had an ORR of $14.7 \%(\mathrm{n}=129)$. A total of $181(73.2 \%)$ patients had either stable disease $(\mathrm{SD})$ or progressive disease (PD). Seventy-one patients $(60.2 \%)$ had $\mathrm{SD} / \mathrm{PD}$ in the $\geq 2$ month group while 110 patients $(85.3 \%)$ had $\mathrm{SD} / \mathrm{PD}$ in the $<2$ month group $(\mathrm{p}<0.001)$.

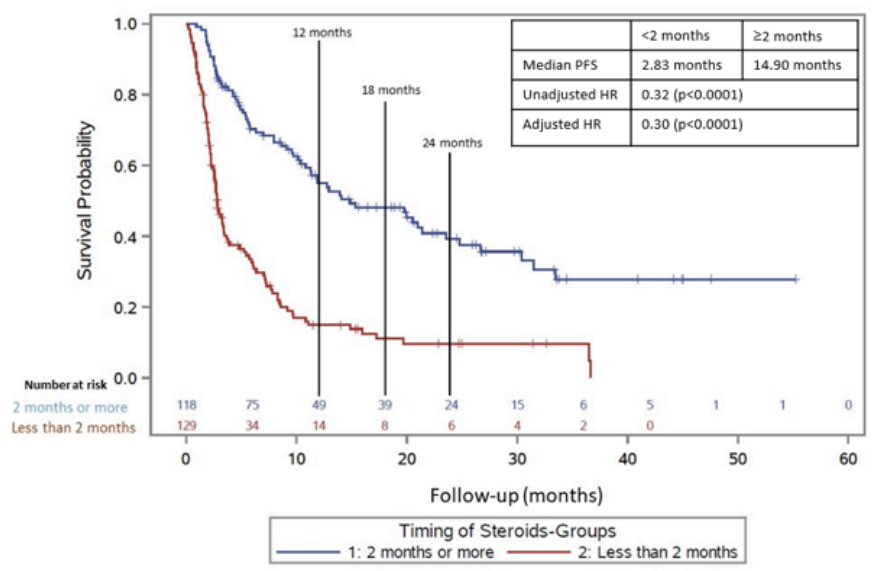

Figure 1 Progression-free survival in those who received $\mathrm{CS} \geq 2$ months after initiation of CPI (blue line) versus those who received CS $<2$ months after initiation of $\mathrm{CPI}$ (red line) in total population. CPI, checkpoint inhibitor; CS, corticosteroid; PFS, progression-free survival.

\section{Differences in outcomes consistent across CS indications}

In a subgroup analysis of only the patients who received CS for irAEs, initiation $\geq 2$ months was associated with an increased PFS compared with $<2$ months (figure 3 ; median PFS 21.0 months vs 4.8 months; adjusted $H R=0.33$; $p$ value $<0.0001)$. For OS, initiation $\geq 2$ months was also associated with an increased OS compared with $<2$ months (figure 4; median OS 31.2 months vs 9.4 months; adjusted $\mathrm{HR}=0.36$; $\mathrm{p}$ value $<0.0001)$.

In a subgroup analysis of only patients who were treated with CS for non-irAE indications, initiation $\geq 2$ months was associated with an increased PFS compared with $<2$ months (figure 5; median PFS 10.8 months vs 2.3 months; adjusted $\mathrm{HR}=0.27$; $\mathrm{p}$ value $<0.0001)$. For $\mathrm{OS}$, initiation $\geq 2$ months was also associated with an increased OS compared with $<2$ months (figure 6; median OS 15.8 months vs 4.3 months; adjusted $\mathrm{HR}=0.31$; $\mathrm{p}$ value $<0.0001$ ).

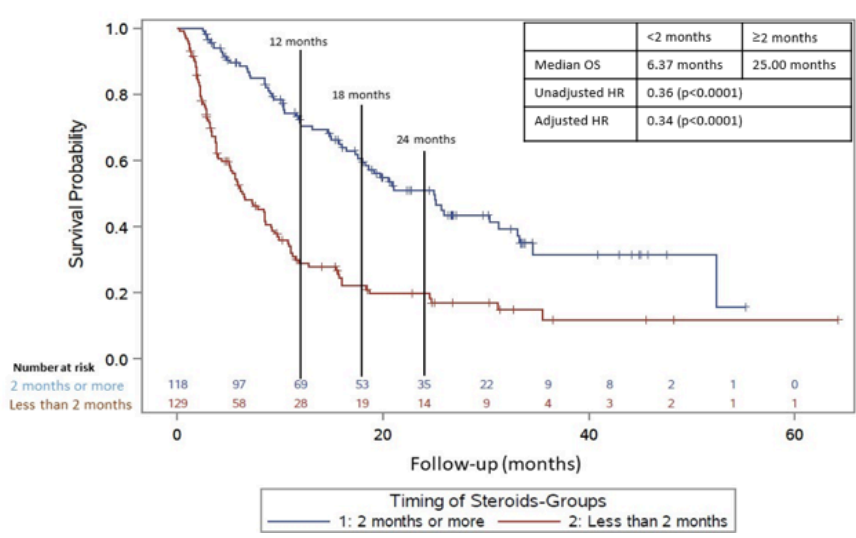

Figure 2 Overall survival in those who received $\mathrm{CS} \geq 2$ months after initiation of CPI (blue line) versus those who received $\mathrm{CS}<2$ months after initiation of $\mathrm{CPI}$ (red line) in total population. CPI, checkpoint inhibitor; CS, corticosteroid; OS, overall survival. 


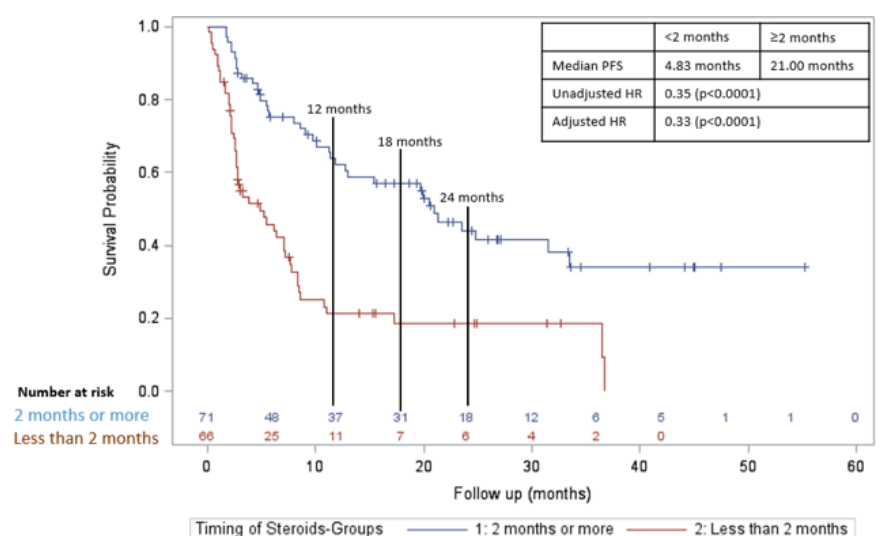

Figure 3 Progression-free survival in those who received $\mathrm{CS} \geq 2$ months after initiation of $\mathrm{CPI}$ (blue line) versus those who received $\mathrm{CS}<2$ months after initiation of $\mathrm{CPI}$ (red line) in those who were treated with CS for irAEs. CPI, checkpoint inhibitor; CS, corticosteroid; irAE, immune-related adverse effect; PFS, progression-free survival.

\section{DISCUSSION/CONCLUSION}

After adjusting for possible confounding factors such as rates of irAEs, drug type, tumor type, and presence of brain metastases, this analysis of 247 patients with cancer who received CS during immune CPI therapy implicates the timing of steroid initiation having a significant impact on outcomes. Patients who received CS $\geq 2$ months after CPI initiation had significantly improved PFS and OS compared with CS initiated $<2$ months. Interestingly we found that, unlike the timing of initiation of CS, higher total steroid dosage did not impact outcomes of patients treated with CPIs.

Many previous studies suggest that CS do not hinder CPI efficacy. Horvat et al studied irAEs caused by ipilimumab, steroid use for irAE management, and effects on OS. They reviewed 254 patients who experienced an irAE from ipilimumab, 103 of which received systemic CS, and 31 required further immunosuppressive therapy. They

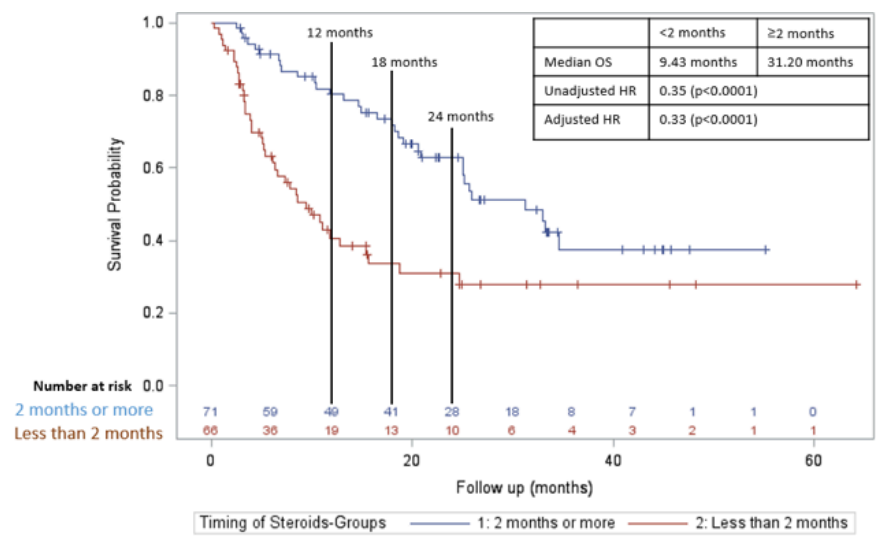

Figure 4 Overall survival in those who received CS $\geq 2$ months after initiation of CPI (blue line) versus those who received $\mathrm{CS}<2$ months after initiation of $\mathrm{CPI}$ (red line) in those who were treated with CS for irAEs. CPI, checkpoint inhibitor; CS, corticosteroid; irAE, immune-related adverse effect; OS, overall survival.

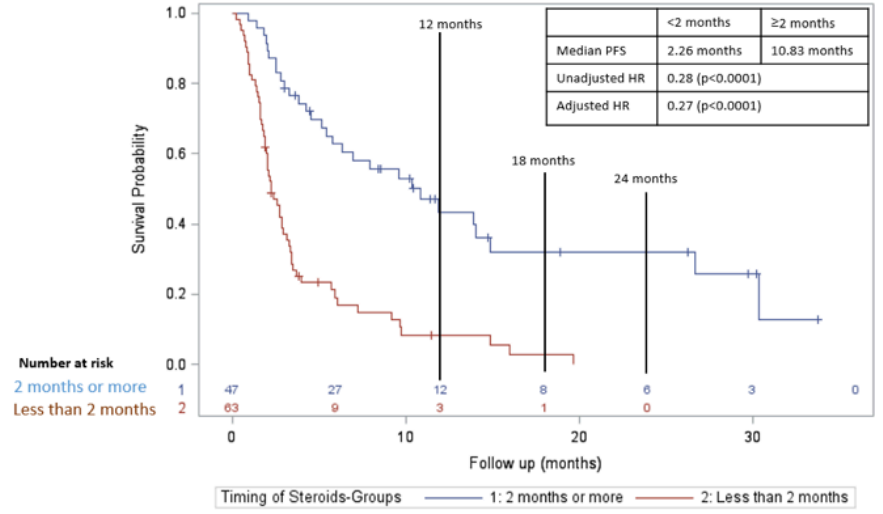

Figure 5 Progression-free survival in those who received $\mathrm{CS} \geq 2$ months after initiation of CPI (blue line) versus those who received $\mathrm{CS}<2$ months after initiation of $\mathrm{CPI}$ (red line) in those who were treated with CS for non-irAEs indications. CPI, checkpoint inhibitor; CS, corticosteroid; irAE, immunerelated adverse effect; PFS, progression-free survival.

observed that there was no difference in time to treatment failure or OS when stratified for administration of systemic CS. ${ }^{13}$ Another study by Garant et al reviewed 155 abstracts comparing patients on immunotherapy who did or did not receive CS also finding that CS did not negatively impact CPI clinical response. ${ }^{14}$ Kapoor et al reviewed 155 patients (38 received CS) and also found no difference in OS between those who received CS and those who did not. ${ }^{15}$ Although these data suggest that use of CS to treat irAEs will not abrogate responses to immunotherapy, they do not support a conclusion that CS does not affect CPI efficacy. Immunotherapy toxicity is associated with higher response rates and improved cancer outcomes from CPIs, and patients that require CS are more likely to have irAEs than patients not requiring CS. We report longer PFS and OS among patients with a documented irAE compared with those without irAEs, which supports this previously reported association of CPI toxicity and improved efficacy. ${ }^{23}$ We also found throughout our subgroup analyses

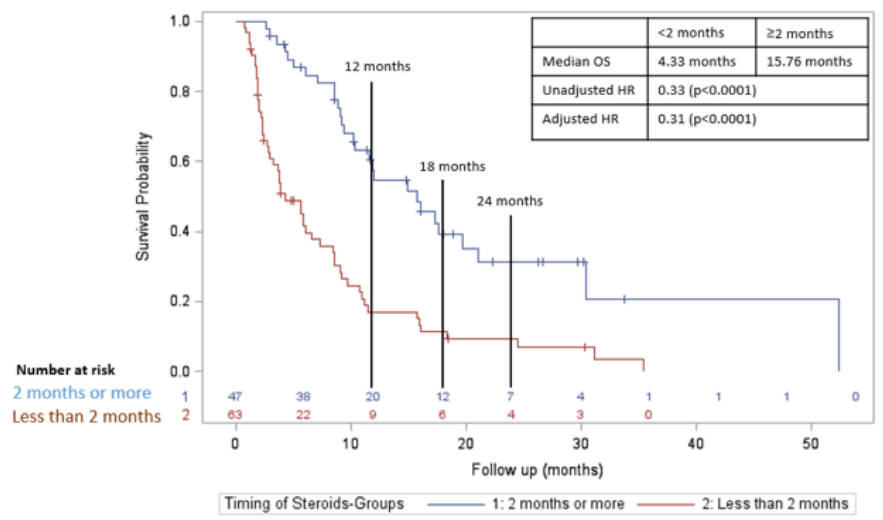

Figure 6 Overall survival in those who received CS $\geq 2$ months after initiation of CPI (blue line) versus those who received $\mathrm{CS}<2$ months after initiation of $\mathrm{CPI}$ (red line) in those who were treated with CS for non-irAEs indications. CPI, checkpoint inhibitor; CS, corticosteroid; irAE, immunerelated adverse effect; OS, overall survival. 
of irAE patients and non-irAE patients, that this assoociation of decreased CPI efficacy with early initiation of CS was consistent across different CS indications.

However, multiple retrospective analyses have found similar results to our study, that there is a negative impact of CS on CPI efficacy, though most of these studies specifically looked at the effects of baseline CS at the time of initiation of CPI. A large systematic review and metaanalysis by Petrelli et al found that a negative impact of steroid use was observed for both OS and PFS in patients that received $\mathrm{CS} .{ }^{18}$ In this review, the negative outcomes were in patients receiving CS for supportive care or brain metastases, while there was no difference in outcomes for patients with irAEs. Potentially factors in these studies affecting prognosis negatively such as brain metastases or palliative care indications may confound conclusions about the direct effects of CS. Similar findings have been described by Ferrara et al., who reviewed patients with NSCLC treated with single-agent PD-L1 blockade, where early steroid use of $\geq 10 \mathrm{mg}$ was associated with poorer outcomes. This study found that patients who received and discontinued CS between days 1 and 30 of CPI initiation had intermediate OS and PFS compared with those who received CS at baseline and those who received no CS. ${ }^{19}$ Consistent with our data, these studies also suggest early CSs have a negative impact on CPI efficacy, but these studies specifically highlight the negative impacts of baseline CS initiated prior to CPI initiation. Also, similar to other studies, these compared patients receiving CS to those not receiving CS. Whether CS initiated later in the course of immune checkpoint blockade has the same effect on cancer outcomes compared with patients receiving earlier CS is unknown.

CS can be immunosuppressive on both the innate and adaptive immune system by inducing effector T-cell apoptosis and impairing maturation of dendritic cells. ${ }^{24}$ CS have also been shown to inhibit the production of important effector T-cell cytokines such as interleukin-2 (IL-2) and interferon-gamma. ${ }^{25}$ There are also preclinical studies describing the mechanisms by which CS preferentially suppress naïve $\mathrm{T}$ cells, which are important for initiation of an anti-tumor immune response. Xing et al have demonstrated in mouse models that dexamethasone enhances PD-1 expression in certain T cell subsets, which would suggest PD-1 blockade might be able to overcome CS T-cell exhaustion. However, this occurred primarily in memory T cells, but naïve T cells did not have a significant increase in PD-1 expression. ${ }^{26}$ Another study by Giles et al found that dexamethasone specifically blocks naïve T-cell proliferation and differentiation by attenuation of CD28 co-stimulation to a greater extent than memory T cells. They also reported in a tumor mouse model that CTLA-4 blockade could partially restore tumor infiltrating interferon gamma producing $\mathrm{T}$ cells in the setting of dexamethasone exposure, but again did not see a significant increase in naïve CD8 T cells. Because co-stimulation is essential for successful T-cell priming and expansion, these preclinical data support the notion that early use of CS may have the greatest impairment of response to immunotherapy. ${ }^{24}$

Although oncologists should be cognizant that early steroid use could reduce CPI efficacy, CS are an important and necessary treatment for certain complications from cancer and from immunotherapy. Along with previously reported data demonstrating inferior outcomes with baseline CS, our results support judicious use of CS early in the course of CPI treatment. ${ }^{19}$ Since we also report in a subgroup analysis inferior outcomes with early CS indicated for the treatment of patients with irAEs, our data highlight the importance of further investigation into steroid sparing strategies using agents that target irAE inflammation (IL-6, Tumor Necrosis Factor (TNF)-alpha, IL-1 inhibitors, etc.). Also when possible, physicians should consider combination chemotherapy/immunotherapy regimens that do not require steroid pretreatments or steroid-sparing anti-emetic regimens that are recommended in National Comprehensive Cancer Network (NCCN) guidelines.

A major limitation of our study is the potential for survivorship bias, an inherent bias of retrospective studies using an independent variable of time. As more patients are collected, enough statistical power can be achieved to perform more in-depth analyses at different CS initiation time points. Future studies should further investigate whether there is an inflection point at which CS do not negatively affect CPI efficacy.

Immune CPIs are used to treat many cancer types. These drugs can lead to irAEs, which often require treatment with CS. Although baseline CS are associated with decreased response rates and outcomes, ${ }^{19}$ more clinical data are needed to evaluate the direct effects of concomitant CS and specifically how the timing of CS affects CPI efficacy. Our study includes only patients with metastatic cancer who were treated with CS while on single-agent or combination CPI therapy to more clearly identify whether timing of initiation of CS directly influences the efficacy of CPIs. We demonstrate that immunosuppression from CS hinders CPI efficacy early in the course of CPI treatment, while late steroid use appears to be less impactful. Delaying CS use and improving steroid-sparing strategies should be considered in patients receiving CPIs.

\section{Twitter Madhav KC @kcmadhav16}

Acknowledgements Thank you to the Ochsner Cancer Center and Department of Statistics for help in data collection throughout this process

Contributors DM, KT and DHJ wrote and edited this manuscript. All other authors helped to contribute with data collection.

Funding The authors have not declared a specific grant for this research from any funding agency in the public, commercial or not-for-profit sectors.

Competing interests There are no competing interests.

Patient consent for publication Not required.

Ethics approval This study was approved by the Ochsner Clinic Foundation Institutional Review Board.

Provenance and peer review Not commissioned; externally peer reviewed.

Data availability statement Data are available upon reasonable request. Data were collected by resident physicians at Ochsner Medical Center through a retrospective chart review. Upon request this can be sent to whom necessary. 
Open access This is an open access article distributed in accordance with the Creative Commons Attribution Non Commercial (CC BY-NC 4.0) license, which permits others to distribute, remix, adapt, build upon this work non-commercially, and license their derivative works on different terms, provided the original work is properly cited, appropriate credit is given, any changes made indicated, and the use is non-commercial. See http://creativecommons.org/licenses/by-nc/4.0/.

\section{ORCID iD}

Diana V Maslov http://orcid.org/0000-0002-3640-3042

\section{REFERENCES}

1 Vaddepally RK, Kharel P, Pandey R, et al. Review of indications of FDA-approved immune checkpoint inhibitors per NCCN guidelines with the level of evidence. Cancers 2020;12:738.

2 Puzanov I, Diab A, Abdallah K, et al. Managing toxicities associated with immune checkpoint inhibitors: consensus recommendations from the Society for immunotherapy of cancer (SITC) toxicity management Working group. J Immunother Cancer 2017;5:95.

3 Sgambato A, Casaluce F, Sacco PC, et al. Anti PD-1 and PDLimmunotherapy in the treatment of advanced non- small cell lung cancer (NSCLC): a review on toxicity profile and its management. Curr Drug Saf 2016;11:62-8.

4 Suresh K, Naidoo J, Lin CT, et al. Immune checkpoint immunotherapy for non-small cell lung cancer: benefits and pulmonary toxicities. Chest 2018;154:1416-23.

5 Tian Y, Abu-Sbeih H, Wang Y. Immune checkpoint Inhibitors-Induced colitis. Adv Exp Med Biol 2018;995:151-7.

6 Mahmood SS, Fradley MG, Cohen JV, et al. Myocarditis in patients treated with immune checkpoint inhibitors. J Am Coll Cardiol 2018;71:1755-64.

7 Shivaji UN, Jeffery L, Gui X, et al. Immune checkpoint inhibitor-associated gastrointestinal and hepatic adverse events and their management. Therap Adv Gastroenterol 2019;12:1756284819884196.

8 Miller S, McNutt L, McCann M-A, et al. Use of corticosteroids for anorexia in palliative medicine: a systematic review. $J$ Palliat Med 2014;17:482-5.

9 Paulsen O, Klepstad P, Rosland JH, et al. Efficacy of methylprednisolone on pain, fatigue, and appetite loss in patients with advanced cancer using opioids: a randomized, placebocontrolled, double-blind trial. J Clin Oncol 2014;32:3221-8.

10 Ryken TC, McDermott M, Robinson PD, et al. The role of steroids in the management of brain metastases: a systematic review and evidence-based clinical practice guideline. J Neurooncol 2010;96:103-14.

11 Ricciuti B, Dahlberg SE, Adeni A, et al. Immune checkpoint inhibitor outcomes for patients with non-small-cell lung cancer receiving baseline corticosteroids for palliative versus Nonpalliative indications. J Clin Oncol 2019;37:1927-34.

12 Carbone DP, Reck M, Paz-Ares L, et al. First-line nivolumab in stage IV or recurrent non-small-cell lung cancer. N Engl J Med 2017;376:2415-26.
13 Horvat TZ, Adel NG, Dang T-O, et al. Immune-Related adverse events, need for systemic immunosuppression, and effects on survival and time to treatment failure in patients with melanoma treated with ipilimumab at Memorial Sloan Kettering cancer center. $J$ Clin Oncol 2015;33:3193-8.

14 Garant A, Vuong T. EP-1384: concomitant use of steroids and immunotherapy in cancer patients: a comprehensive review. Radiotherapy and Oncology 2017;123:S741-2.

15 Kapoor A, Noronha V, Patil V. Concomitant use of corticosteroids and immune checkpoint inhibitors in patients with solid neoplasms: a real-world experience from a tertiary cancer center. Cancer Res Stat 2019;2:204-8.

16 Okoye IS, Xu L, Walker J, et al. The glucocorticoids prednisone and dexamethasone differentially modulate $\mathrm{T}$ cell function in response to anti-PD-1 and anti-CTLA-4 immune checkpoint blockage. Cancer Immunol Immunother 2020;69:1423-36.

17 Harmankaya K, Erasim C, Koelblinger C, et al. Continuous systemic corticosteroids do not affect the ongoing regression of metastatic melanoma for more than two years following ipilimumab therapy. Med Oncol 2011;28:1140-4.

18 Petrelli F, Signorelli D, Ghidini M, et al. Association of steroids use with survival in patients treated with immune checkpoint inhibitors: a systematic review and meta-analysis. Cancers 2020;12:546.

19 Arbour KC, Mezquita L, Long N, et al. Impact of baseline steroids on efficacy of programmed cell death-1 and programmed Death-Ligand 1 blockade in patients with non-small-cell lung cancer. $J$ Clin Oncol 2018;36:2872-8.

20 Fucà G, Galli G, Poggi M, et al. Modulation of peripheral blood immune cells by early use of steroids and its association with clinical outcomes in patients with metastatic non-small cell lung cancer treated with immune checkpoint inhibitors. ESMO Open 2019;4:e000457.

21 Scott SC, Pennell NA. Early use of systemic corticosteroids in patients with advanced NSCLC treated with nivolumab. J Thorac Oncol 2018;13:1771-5.

22 De Giglio A, Mezquita L, Auclin E, et al. Impact of intercurrent introduction of steroids on clinical outcomes in advanced nonsmall-cell lung cancer (NSCLC) patients under Immune-Checkpoint inhibitors (ICI). Cancers;12:2827.

23 Owen DH, Wei L, Villalona-Calero MA, et al. Impact of immunerelated adverse events (irAE) on overall survival (OS) in patients treated with immunotherapy for non-small cell lung cancer (NSCLC). JCO 2017;35:9080.

24 Giles AJ, Hutchinson M-KND, Sonnemann HM, et al. Dexamethasone-Induced immunosuppression: mechanisms and implications for immunotherapy. J Immunother Cancer 2018;6:51.

25 Almawi WY, Beyhum HN, Rahme AA, et al. Regulation of cytokine and cytokine receptor expression by glucocorticoids. J Leukoc Biol 1996;60:563-72.

26 Xing K, Gu B, Zhang P, et al. Dexamethasone enhances programmed cell death 1 (PD-1) expression during $T$ cell activation: an insight into the optimum application of glucocorticoids in anti-cancer therapy. BMC Immunol 2015;16:1-9. 